\section{Entropic Analysis of HRV in Obese Children}

Franciele M. Vanderlei ${ }^{1}$, Luiz Carlos M. Vanderlei ${ }^{1}$, Luiz Carlos de Abreu², David M. Garner ${ }^{3}$

\title{
Abstract
}

The aim of the study was to analyze heart rate dynamics in obese children by functional entropic measures of Heart Rate Variability (HRV). HRV is a simple, reliable, cheap and non-invasive measure of autonomic impulses. We applied five technques based on entropy to assess the level of complexity. These were Shannon, Multiscale Tsallis and Multiscale Rényi entropies. Then, Approximate and Sample entropies. Ninety-four children of mixed gender aged eight to twelve years were divided into two equal groups $(n=47)$ based on body mass index: obese and non-obese weight ranges. HRV was monitored in the dorsal decubitus position for 20 minutes. After Anderson-Darling and Ryan-Joiner tests of normality, the parametric test ANOVA1 was applied for the statistical analysis, with the level of significance set at $(p<0.05)$; so the probability of a type I error was less than $5 \%$. All types of functional entropies were significant at that level with the exception of Sample entropy. Furthermore, for all five measures the chaotic response increased when undergoing change from non-obese to obese. Regarding the application of Principal Component Analysis (PCA) the first two components represent $98.9 \%$ of total variance; a steep scree plot. The Multiscale Rényi $(\alpha=0.25)$, Shannon and Multiscale Tsallis $(q=0.25)$ entropies performed simlarly regarding PCA and ANOVA1; whilst the Approximate and Sample entropies were also analogous with respect to these particular statisical tests. The Approximate entropy performed the most strongly with respect to $p$-value $(p=0.0092)$ by ANOVA1 and PCA. With the exception of Sample entropy the entropic techniques described here were able to significantly quantify the increase in chaotic response when non-obese to obese children were assessed by the HRV.

\section{Keywords}

Principal Component Analysis; Electrocardiography; Rényi Entropy;

Tsallis Entropy; Obesity.

1 Department of Physiotherapy, UNESP - Univ Estadual Paulista- Presidente Prudente, Sao Paulo, Brazil.

2 Laboratório de delineamento de Estudos e Escrita Científica. Disciplina de metodologia Científica. Faculdade de Medicina do ABC, FMABC, Santo André, Brazil.

3 Cardiorespiratory Research Group,Department of Biological and Medical Sciences, Faculty of Health and Life Sciences,Oxford Brookes University, Gipsy Lane, Oxford OX3 OBP, United Kingdom.

\section{Contact information:}

\section{Luiz Carlos de Abreu.}

Laboratório de delineamento de Estudos e Escrita Científica. Disciplina de metodologia Científica.

Faculdade de Medicina do ABC, FMABC, Santo André, Brazil.

झ luiz.abreu@fmabc.br 


\section{Introduction}

Investigation of asymmetrical changes have been rigourously investigated in the life and biomedical sciences [1-4]. Various functional entropies enforce data of interpeak intervals to characterize irregular and often chaotic variation. They are Multiscale Tsallis [5], Multiscale Rényi [6], Shannon [7], Sample [8] and Approximate [9] entropies. This is widely accepted for Electrocardiograph (ECG) traces where the temporal separations or RR-interval of the PQRST-signature are suitable. The autonomic nervous system (ANS) regulates heart rate through sympathetic and parasympathetic branches. Broadly speaking, the sympathetic activity increases heart rate and decreases Heart Rate Variability (HRV); the parasympathetic activity decreases heart rate and increases HRV [10].

Obesity is a major public health problem [11]. Consequences include arterial hypertension, atherosclerosis, diabetes, sleep apnea and depression; amongst others. Studies have revealed that obesity produces abnormalities of the ANS in children [12] and young adults [13]. Autonomic behaviour is assessed by HRV a simple, inexpensive and noninvasive tool for the detection and study of cardiac irregularities in many dynamical conditions. However the perceived benefit for testing the correlation with HRV is that it can provide an indicator of the risk of cardiac failure and other "dynamical diseases [14]" in such children. This is a consequence of mechanisms involved in cardiovascular regulation that interact with each other in a complex and chaotic manner. The aim of this is to analyze heart rate dynamics in obese children by functional entropic measures of HRV.

\section{Methods}

\section{Population}

A total of 94 subjects of mixed gender between eight and twelve years of age were divided into two equal groups $(n=47)$ based on body mass index: obese and non-obese. Obesity was defined on body mass index established for age and gender. Children reported using drugs or diagnosed diseases were not included in this study. The volunteers and parents/guardians were duly informed as to the procedures and objectives of the study and, after agreeing to participate, the parents/guardians signed terms of informed consent. All procedures received approval from the ethics committee of the Faculdade de Ciências e Tecnologia - FCT/UNESP (Process no 187/2007).

\section{Experimental protocol}

Prior to beginning the experimental procedure, demographic and other information was collected on age, gender, weight, height and body mass index. Weight was determined using a digital scale (Filizzola PL 150, Filizzola Ltda., Brazil) with a precision of $0.1 \mathrm{~kg}$, with the children barefoot and wearing light-weight clothing. Height was determined using a stadiometer with a precision of $0.1 \mathrm{~cm}$.

The data collection was undertaken in a room with the temperature $\left(21\right.$ to $23{ }^{\circ} \mathrm{C}$ ) and, relative humidity (40 to 60\%). Data were collected between 14:00 and 17:00 to minimize circadian rhythm interference.

After the initial evaluation, all procedures necessary for the data collection were explained on an individual basis and the children were instructed to remain at rest and avoid talking during the collection. The heart monitor belt was then placed over the thorax, aligned with the distal third of the sternum and the Polar S810i heart rate receiver (Polar Electro, Finland) was placed on the wrist. The equipment was previously validated for monitoring beat-by-beat heart rate and the use of these data for HRV analysis [15]. The children were placed in the dorsal decubitus position and remained at rest with spontaneous breathing for 20 minutes. After the collection, the child was discharged. The HRV behaviour pattern was re- 
corded beat-by-beat throughout the monitoring process at a sampling rate of $1000 \mathrm{~Hz}$. Following digital filtering complemented with manual filtering for the elimination of premature ectopic beats and artifacts, 1000 consecutive interbeat intervals were used for the data analysis. Only series with more than 95\% sinus rhythm was included in the study [16].

\section{Entropic Categories}

From the information theory point of view, entropy is a benchmark of the disorder in dynamical systems, a statistical complexity measurement. Entropy-based techniques are routinely employed in analysis of medical data especially cardiovascular [17-19], respiratory $[20,21]$ and neurological signals $[22,23]$. In this case, the cardiovascular system that composes the dynamical systems was scrutinized in obese and non-obese children.

\section{Shannon Entropy}

Generally, entropy as a measure of lack of knowledge is useful in many situations. For conditions where the connection with physical temperature is unimportant, the Boltzmann's constant can be removed by normalizing the entropy with the constant $k s=1 / \log (2)$. This normalisation gives us the Shannon entropy, $\mathrm{S}_{\mathrm{s}}[\mathrm{P}]$ [24]. A low entropy dataset is highly predictable - whereas a high entropy dataset is less predictable. So, high entropy is more disordered or random. $\mathrm{P} \equiv\left\{\mathrm{p}_{1}, \ldots \ldots \ldots . ., \mathrm{p}_{\mathrm{N}}\right\}$ is the time series

$$
S_{s}[P]=\sum_{j=1}^{N} p_{j} \ln p_{j}
$$

In contrast to other Rényi entropies; Shannon entropy is additive. So if the probabilities can be factorised into independent factors, the entropy of the joint process is the sum of the entropies of the separate processes.

\section{Multiscale Rényi Entropy}

The order-q Rényi entropies is a series of entropy like quantities. Taking the limit $\alpha \rightarrow 1$, Rényi entropy coincides with Shannon entropy; which can be derived by the de I'Hospital rule. Here we set the value, $\alpha$ to $0.25,0.35,0.45,0.55,0.65,0.75 . \mathrm{P} \equiv$ $\left\{\mathrm{p}_{1}, \ldots \ldots \ldots . ., \mathrm{p}_{\mathrm{N}}\right\}$ is the time series and, $\alpha$ the entropy order. Where $\alpha=1$ the function is the Shannon entropy and when $\alpha=2$ it is the squared entropy. When $\alpha$ is varied this provides the multi-scale measure; $\alpha=0$ is simply the logarithm of $n$. As $\alpha$ is increasing the measures become more sensitive to the values occurring at a higher probability and less to those at a lower probability. Thus, an image of the RR-interval length distribution within a signal is provided.

$$
S_{R}\left([\alpha,[P])=\frac{1}{1-\alpha} \ln \left[\sum_{j=1}^{N} p_{j}^{\alpha}\right]\right.
$$

\section{Multiscale Tsallis Entropy}

Tsallis entropy is a generalization of the standard Shannon-Boltzmann-Gibbs entropy. It was introduced as a basis for generalizing the standard statistical mechanics. It is non-additive entropy. The limit $q \rightarrow 1$ leads to Tsallis entropy to coincide with the Shannon-Boltzmann-Gibbs entropy. Here we set $q$ to $0.25,0.35,0.45,0.55,0.65,0.75$. $\mathrm{P} \equiv$ $\left\{\mathrm{p}_{1}, \ldots \ldots \ldots \ldots, \mathrm{p}_{\mathrm{N}}\right\}$ is the time series and, $\mathrm{q}$ is the entropic index. Where $q=1$ it is the Shannon-BoltzmannGibbs entropy.

$$
S_{T}\left([q,[P])=\frac{1}{q-1} \sum_{j=1}^{N}\left[p_{j} \ln p_{j}^{q}\right]\right.
$$

\section{Approximate Entropy}

Approximate entropy is the logarithmic ratio of component wise matching sequences from the signal length, $N$. Other relevant parameters involve $r$ which we set to 0.2 of the standard deviation based on factors of the signal that is being analyzed 
and compared to. The factor $m$, is the length of sequences compared which we set to window of 2. It is measured as an integer count of discrete time bins. A minimum value of zero for Approximate entropy would indicate a fully predictable series. Approximate entropy is described algorithmically as follows [25].

Given $\mathrm{N}$ data points from a time-series $\{\mathrm{x}(\mathrm{n})\}=$ $x(1), x(2), \ldots . . x(N)$, one should follow these steps to compute Approximate entropy:

Form $N-m+1$ vectors $X(1), X(2), \ldots \ldots \ldots, X(N-m+1)$ defined by $X(\mathrm{i})=[x(\mathrm{i}), x(\mathrm{i}+1), \ldots . ., x(i+m-1)], i=1, \ldots ., N-m+1$.

These vectors represent $m$ consecutive $x$ values, beginning with the $i^{\text {th }}$ point.

Define the distances between $X(\mathrm{i})$ and $X(\mathrm{j}), d[X(\mathrm{i})$, $X(\mathrm{j})$ ], as the maximum norm,

$$
d[X(i), X(j)]=\max _{k=1, \ldots, m}(|x(i+k-1)-x(j+k-1)|)
$$

For a given $X(\mathrm{i})$, count the number of $j(\mathrm{j}=1, \ldots .$. , $N-m+1)$, so that $\mathrm{d}[X(\mathrm{i}), X(\mathrm{j})] \leq \mathrm{r}$, denoted as $N^{m}(i)$, then, for $i=1, \ldots . N-m+1$,

$$
C_{r}^{m}(i)=\frac{N^{m}(i)}{N-m+1}
$$

with a tolerance $r$, the frequency of patterns similar to a given one of window length $m$.

Compute the natural logarithm of each $C_{r}^{m}(i)$ and average it over $i$,

$$
\phi^{m}(r)=\frac{1}{N-m+1} \sum_{i=1}^{N-m+1} \ln C_{r}^{m}(i)
$$

Increase the dimension to $m+1$. Repeat steps 1-4 and find and $\phi^{m+1}(r)$

Approximate entropy is defined as

$$
\operatorname{ApEn}(m, r, N)=\phi^{m}(r)-\phi^{m+1}(r)
$$

\section{Sample Entropy}

It is important to consider Approximate entropy and Sample entropy together as similar mathematical functions. Comparisions with fixed $m, r$, and $N$. $N$ is the length of the time series and $m$ is the length of the sequences to be compared whereas $r$ is the tolerance for accepting matches. As with Approximate entropy in this study we set $r$ to 0.2 of the standard deviation. The factor $m$, is the length of sequences compared which we set to window of 2. The algorithm for Sample entropy is discussed below mathematically [25].

Given $N$ data points from a time series $\{\mathrm{x}(\mathrm{n})\}=\mathrm{x}(1), \mathrm{x}(2), \ldots \ldots \mathrm{x}(\mathrm{N})$.

Form $m$ - vectors $X_{m}(1), X_{m}(2) \ldots X_{m}(N-m+1)$; the distance between $X_{m}(i)$ and $X_{m}(j)$ is defined as:

$$
d[X(i), X(j)]=\max _{k=1, \ldots, m}(|x(i+k-1)-x(j+k-1)|)
$$

For a given $X_{m}(i)$, count the number of $j(1 \leq j \leq$ $N-m, j \neq i$ ) denoted as $B_{i}$, such that the distance between $X_{m}(i)$ and $X_{m}(j)$ is less than or equal to $r$.

Then, $1 \leq i \leq N-m$,

$$
\begin{aligned}
& B_{i}^{m}(r)=\frac{1}{N-m-1} B_{i} \\
& \text { with, } \\
& B^{m}(r)=\frac{1}{N-m} \sum_{i=1}^{N-m} B_{i}^{m}(r),
\end{aligned}
$$

We increase the dimension to $m+1$ and calculate $A_{i}$ as the number of $X_{m}+1(i)$ within $r$ of $X_{m}+1(j), j$ is from 1 to $N-m(j \neq i)$.

We then define

$$
A_{i}^{m}(r)=\frac{1}{N-m-1} A_{i}
$$

Where

$$
A^{m}(r)=\frac{1}{N-m} \sum_{i=1}^{N-m} A_{i}^{m}(r)
$$

$B^{m}(r)$ is the probability that two sequences will match for $m$ points $A^{m}(r)$ is the probability that two sequences will match for $m+1$ points. 
Sample entropy is defined as

$$
\operatorname{SampEn}(m, r, N)=-\ln \left[\frac{A^{m}(r)}{B^{m}(r)}\right]
$$

Figure 1: A time-series of ECG interpeak temporal separations (RR beat durations) for an obese child. For the chaotic parameters (i) Shannon entropy (ii) Multiscale Rényi entropy (various a) (iii) Multiscale Tsallis entropy (various q) (iv) Approximate entropy and (v) Sample entropy. The algorithms are applied directly to the RR beat durations. No power spectrum is generated or applied in any way to evaluate these measurements.

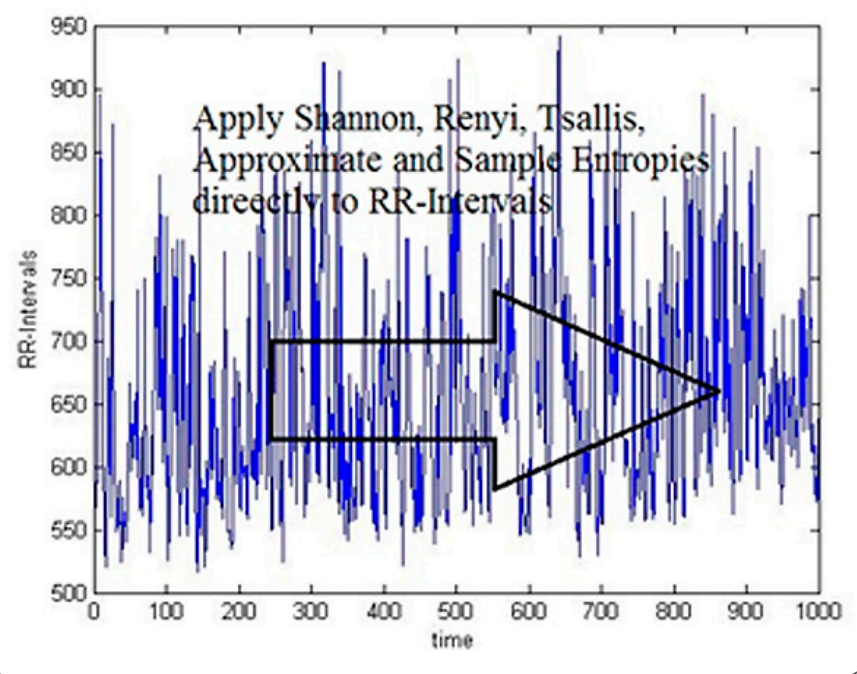

\section{Results}

\section{Mean Variation \& Significances}

Parametric statistics generally assume the data are normally distributed, hence the use of the mean as a measure of central tendancy. If we cannot normalize the data we should not compare means. To test our assumptions of normality we applied the Anderson-Darling [26] and Ryan-Joiner tests [27] of normality. The Anderson-Darling test for normality applied an empirical cumulative distribution function, whereas the Ryan-Joiner test is a correlation based test similar to Shapiro-Wilk test [28]. In the majority of cases the $p>0.1$; so we can say that the observations follow a normal distribution. Therefore we have a probability plot of mainly normal data and we applied the one-way analysis of variance (ANOVA1) ; the parametric test of significance.

The results illustrate that there is a wide variation in both the mean values for both obese and non-obese (See Table 1). The p-value calculated is the ANOVA1 parameter. The algorithm computes a significant statistical result for four of the five combinations with the probability of a type I error was less than $5 \%(p<0.05)$. These are all combinations except Sample entropy - Sample entropy is significant at $p<0.1$. For all five measurements the chaotic response increased from non-obese to obese. Both Multiscale Rényi and Multiscale Tsallis entropy were most significant at $\alpha=0.25$ and $q=0.25$. In the next section regarding the multivariate analysis (Section 4.2) we only observe the Rényi and Tsallis entropies at these optmium values.

Table 1. The table below shows the mean values, standard deviation and $p$-value of significance for the five entropic measures for non-obese and obese subjects when related to RR-intervals. The number of RRintervals was 1000; and ANOVA1 test of significance applied. For Multiscale Rényi and Multiscale Tsallis entropy the values we calculated were for six values of entropic order and entropic index. For Approximate entropy and Sample entropy $(m=2$ and $r=0.2$ ).

\begin{tabular}{|c|c|c|c|}
\hline & Mean \pm SD & Mean \pm SD & ANOVA1 \\
\hline Param & $\begin{array}{c}\text { Non-Obese } \\
(n=47)\end{array}$ & $\begin{array}{l}\text { Obese } \\
(n=47)\end{array}$ & (p-valu \\
\hline Approx & $0.7842 \pm 0.1205$ & $0.8464 \pm 0.1059$ & 0.0092 \\
\hline Sample & $0.7278 \pm 0.1242$ & $0.7744 \pm 0.1204$ & $0 \cap 88$ \\
\hline Shannon & $0.6381 \pm 0.0988$ & $0.6937 \pm 0.1811$ & 0.0151 \\
\hline
\end{tabular}




\begin{tabular}{|c|c|c|c|}
\hline \multirow{2}{*}{$\begin{array}{c}\text { Entropic } \\
\text { Parameter }\end{array}$} & Mean \pm SD & Mean \pm SD & ANOVA1 \\
\hline & $\begin{array}{c}\text { Non-Obese } \\
(n=47)\end{array}$ & $\begin{array}{l}\text { Obese } \\
(n=47)\end{array}$ & (p-value) \\
\hline \multicolumn{4}{|l|}{ Rényi } \\
\hline$\alpha=0.25$ & $0.9884 \pm 0.0037$ & $0.9905 \pm 0.0042$ & 0.0126 \\
\hline$\alpha=0.35$ & $0.9849 \pm 0.0049$ & $0.9876 \pm 0.0055$ & 0.0132 \\
\hline$\alpha=0.45$ & $0.9819 \pm 0.0058$ & $0.9852 \pm 0.0066$ & 0.0138 \\
\hline$\alpha=0.55$ & $0.9794 \pm 0.0067$ & $0.9830 \pm 0.0075$ & 0.0144 \\
\hline$\alpha=0.65$ & $0.9771 \pm 0.0074$ & $0.9811 \pm 0.0084$ & 0.0149 \\
\hline$\alpha=0.75$ & $0.9751 \pm 0.0080$ & $0.9795 \pm 0.0091$ & 0.0155 \\
\hline \multicolumn{4}{|l|}{ Tsallis } \\
\hline$q=0.25$ & $0.6741 \pm 0.0906$ & $0.7254 \pm 0.1075$ & 0.0141 \\
\hline$q=0.35$ & $0.6732 \pm 0.0908$ & $0.7246 \pm 0.1078$ & 0.0142 \\
\hline$q=0.45$ & $0.6718 \pm 0.0912$ & $0.7233 \pm 0.1083$ & 0.0143 \\
\hline$q=0.55$ & $0.6694 \pm 0.0917$ & $0.7213 \pm 0.1090$ & 0.0144 \\
\hline$q=0.65$ & $0.6659 \pm 0.0926$ & $0.7182 \pm 0.1101$ & 0.0145 \\
\hline$q=0.75$ & $0.6607 \pm 0.0938$ & $0.7136 \pm 0.1116$ & 0.0146 \\
\hline
\end{tabular}

Table 2. The table below is the Principal Component Analysis for five groups of entropy for fourty-seven subjects who are obese children. PC1 represents the First Principal Component, PC2 the Second; until the fifth component PC5. For Rényi and Tsallis entropy the values we calculated for the optimum values of entropic order $(\alpha=0.25)$ and entropic index $(q=0.25)$. For Approximate entropy and Sample entropy $(m=2 ; r=0.2)$.

\begin{tabular}{|c|c|c|c|c|c|}
\hline $\begin{array}{l}\text { Entropic } \\
\text { Parameter }\end{array}$ & PC1 & PC2 & PC3 & PC4 & PC5 \\
\hline Approximate & 0.402 & -0.621 & -0.673 & 0.027 & $<0.001$ \\
\hline Sample & 0.427 & -0.523 & 0.737 & -0.019 & $<0.001$ \\
\hline Shannon & 0.467 & 0.341 & -0.016 & 0.477 & 0.661 \\
\hline \multicolumn{6}{|l|}{ Rényi } \\
\hline$\alpha=0.25$ & 0.468 & 0.330 & -0.057 & -0.813 & 0.084 \\
\hline \multicolumn{6}{|l|}{ Tsallis } \\
\hline$q=0.25$ & 0.468 & 0.340 & -0.021 & 0.331 & -0.745 \\
\hline
\end{tabular}

\section{Principal Component Analysis (PCA)}

Principal Component Analysis [29] (See Table 2) is a multivariate statistical procedure where the random observations are transformed into a smaller set of uncorrelated variables called Principal Components (PCs). In other words, the original data is presented as a weighted sum of orthagonal basis vectors; where the basis vectors are the eigenvalues of data covariance or correlation matrix and the weights are the PCs. Typical applications of PCA included data reduction, feature extraction and visualization of multidimensional data.

We had the values of five groups for fourty-seven subjects who are obese; hence a grid of 5-by- 47 to be assessed. The First Principal Component had a variance (eigenvalue) of 4.1406 and accounted for $82.8 \%$ of the total variance. The Second Principal Component had an eigenvalue of 0.8034 accounted for $98.9 \%$ of total variance. The second component had a proportion of influence of $16.1 \%$. Therefore we assumed that most variance was acheived in the first two components; a steep scree plot.

Taking the principal components into account we can see that the Shannon, Rényi $(\alpha=0.25)$ and Tsallis $(q=0.25)$ entropies have very similar first and second principal components (PC1 \& PC2). Whereas, the Approximate and Sample entropy are correspondingly grouped with similar first and second principal components (PC1 \& PC2). Most of the variance is achieved within the first two components and so we need not discuss third (PC3) to fifth (PC5) principal components cited in Table 2. We therefore represent the HRV data using the first two PCs corresponding to the most significant eigenvectors.

\section{Discussion}

Algorithmic development to characterise HRV of healthy and unhealthy subjects is an ongoing event especially to nonlinear measures, as standard measures such as SDNN can lead to incorrect interpretation of pathology. Usually in physiology a high 
HRV is a signal of good adaptation and characterize a healthy person with efficient autonomic mechanisms. Although low HRV is frequently an indicator of abnormal and insufficient adaptation of the ANS; causing the subject low physiological function. However, here there has been an increase in chaotic response when going from non-obese to obese children.

Regarding Shannon, Rényi $(\alpha=0.25)$ and Tsallis $(q=0.25)$ entropies the results are very similar with regards to ANOVA1 and PCA. It is also the case that these three parameters respond to chaos in the same way increasing their responses when going from non-obese to obese. It is usually the case that pathological conditions such as obesity would decrease their chaotic responses; but this is not the case here.

Approximate entropy is a technique applied to quantify the amount of regularity and the unpredictability of fluctuations over time-series data. The creation of Approximate entropy was motivated by the search for a distribution free measure of regularity. Unlike Shannon entropy, Approximate entropy is not predicated on the underlying distribution of the data. But instead relies on sequence recurrences. Consequently it is more suitable to signals of shorter length. In addition it makes the use of model estimation invalid removing the liability for false estimation based on poor model selection. It was developed as a tool that could be applied to both correlated random and noisy deterministic processes, and its motivation is drawn from the fields of nonlinear dynamics and chaos theory. In particular, the entropy being approximated is Kolmogorov-Sinai Entropy, which is generally finite for deterministic processes and infinite for random processes.

Advantages of Approximate Entropy include:

a) Low computational demand

b) Applied online in a clinical setting and retrospectively in the laboratory.

c) Decipher the information in the presence of significant noise level.
Disadvantage of Approximate Entropy technique:

a) Very dependent on the parameters choice.

b) There is a certain bias to the technique (see below: Sample Entropy)

c) No guideline available for the values; run length $m$ and, tolerance window $r$

These disadvantages makes the results from Approximate entropy especially difficult to interpret and is therefore only best consulted in combination with other methods such as Sample entropy, which then helps understand the datasets response. The Approximate entropy algorithm counts each sequence as matching itself to avoid the occurance of $\ln (0)$ in the calculations; which is why bias can be problematic. Sample entropy is the negative logatithm of the conditional probability that two sequences similar to $m$ points remain similar at the next point, where self-matches are not included in calculating the probability.

Sample entropy and Approximate entropy perform similary with respect to PCA. Nevertheless, only Sample entropy was insignificant at the level $5 \%$ ( $p>0.05)$ In Sample entropy the number of matches can be increased by choosing small templates and wide tolerances. For smaller tolerances one usually achieves poor conditional probability estimates while for larger tolerances too much detailed system information is lost.

Advantages of Sample Entropy include:

a) Attempts reduce the bias found in Approximate entropy.

b) Lower values indicate more self-similarity in the time-series than Approximate entropy.

c) More statistically robust than Approximate entropy.

d) Algorithm is simpler and eliminates self matches.

Disadvantages of Sample Entropy include:

a) It is highly independent of dataset length

b) No directive available for tolerance window $r$ or run length $m$. 


\section{Conclusion}

Traditional HRV analysis is founded on the basis of linearity, stationarity and equilibrium of fluctuating heart rate time signals. Nonlinear and multiscale studies have indicated a high level of complexity in HRV dynamics based on the observation of a power law relationship when viewing the power spectral density for varying time scales. The traditional methods are insufficient when attempting to quantify the complex dynamics found in oscillating heart rate time series (RR-intervals of the ECG).

With the exception of Sample entropy the entropic techniques described here were able to significantly quantify the increase in chaotic response when non-obese to obese children were assessed by the HRV. It is noted that this is contrary to the usual results in the sense that pathological conditions usually decrease the complexity of HRV. Here the problem obesity increases the chaotic reaction in HRV. There have been studies in the past which have exibited such as response - studies in child and youth obesity. We conclude that obesity is a novel condition with regards to its chaotic response. Such a response is not usually found in "dynamical diseases."

HRV entropy measures; with the exception of Sample entropy can be used for assessing the risk of dynamical diseases due to HRV in obese children. This is especially the case for Multiscale Rényi and Tsallis entropies with the appropriate values of entropic order, $\alpha$ and entropic index, q respectively. Nevertheless, Approximate entropy remains the favoured choice as its significance by its ANOVA1 pvalue is clearly most noteworthy at $p=0.0092$.

\section{Conflict of Interests}

The authors declare that there is no conflict of interests regarding the publication of this article

\section{Acknowledgements}

Funded by the Foundation of Support to Research of Sao Paulo State - FAPESP grant 2008/05414-0 and

\section{References}

1. Costa MD, Henriques $T$, Munshi MN, Segal AR, Goldberger AL. Dynamical glucometry: Use of multiscale entropy analysis in diabetes. Chaos. 2014; 24(3): 033139. DOI: http://dx.doi. org/10.1063/1.4894537.

2. Goldberger AL, Rigney $D R$, West $B J$. Chaos and fractals in human physiology. Sci Am. 1990; 262(2): 42-9.

3. Goldberger AL, West BJ. Chaos and order in the human body. MD Comput. 1992; 9(1): 25-34

4. Seely AJE, Macklem PT. Complex systems and the technology of variability analysis. Crit Care. 2004; 8(6): R367-84.

5. Silva LEV, Murta LO. Evaluation of physiologic complexity in time series using generalized sample entropy and surrogate data analysis. Chaos. 2012; 22(4): 043105. DOI: http://dx.doi. org/10.1063/1.4758815.

6. Zyczkowski K. Renyi extrapolation of Shannon entropy. Open Sys Inf Dyn. 2003; 10(3): 297-310. DOI: http://dx.doi. org/10.1023/A:1025128024427.

7. Shannon CE. A mathematical theory of communication. Bell Sys Tec J. 1948; 27(3): 379-423. DOI: http://dx.doi. org/10.1002/j.1538-7305.1948.tb01338.x

8. Richman JS, Lake DE, Moorman JR. Sample entropy. Methods Enzymol. 2004; 384: 172-84. DOI: http://dx.doi.org/10.1016/ S0076-6879(04)84011-4.

9. Pincus S. Approximate entropy (ApEn) as a complexity measure. Chaos. 1995; 5(1): 110-7. DOI: http://dx.doi. org/10.1063/1.166092.

10. Berntson GG, Bigger JT Jr, Eckberg DL, Grossman P, Kaufmann $P G$, Malik $M$, et al. Heart rate variability: origins, methods, and interpretive caveats. Psychophysiology. 1997; 34(6): 623-48. DOI: http://dx.doi.org/10.1111/j.1469-8986.1997.tb02140.x

11. Vanderlei FM, Vanderlei LC, Garner DM. Chaotic global parameters correlation with heart rate variability in obese children. Journal of Human Growth and Development. 2014; 24(1): 24-30

12. Raimundo RD, Godleski JJ. Heart rate variability in metabolic syndrome. Journal of Human Growth and Development. 2015; 25(1): 7-10. DOI: http://dx.doi.org/10.7322/jhgd.96757

13. Vanderlei FM, Vanderlei LCM, Garner DM. Heart rate dynamics by novel chaotic globals to HRV in obese youths. Journal of Human Growth and Development. 2015; 25(1): 82-88. DOI: http://dx.doi.org/10.7322/jhgd.96772. 
14. Mackey CM, Milton JG. Dynamical diseases. Ann New York Acad Sci. 1987; 504(1): 16-32. DOI: http://dx.doi. org/10.1111/j.1749-6632.1987.tb48723.x.

15. Vanderlei LCM, Silva RA, Pastre CM, Azevedo FM, Godoy MF. Comparison of the Polar S810i monitor and the ECG for the analysis of heart rate variability in the time and frequency domains. Braz J Med Biol Res. 2008; 41(10): 854-9. DOI: http://dx.doi.org/10.1590/S0100-879X2008005000039.

16. Godoy MF, Takakura IT, Correa PR. Relevância da análise do comportamento dinâmico não linear (Teoria do Caos) como elemento prognóstico de morbidade e mortalidade em pacientes submetidos à cirurgia de revascularização miocárdica. Arq Ciênc Saúde. 2005; 12(4): 167-71.

17. Bernardo AFB, Vanderlei LCM, Garner DM. HRV Analysis -A clinical and diagnostic tool in Chronic Obstructive Pulmonary Disease. Int Schol Res Notices. 2014; 2014: 1-6. DOI: http://dx.doi.org/10.1155/2014/673232.

18. Fontes AMG, Garner DM, Abreu LC, Barbosa JC, Assis EV, Souza ACA, et al. Global chaotic parameters of heart rate variability during mental task. Complexity. 2015; 00(00): 1-8. DOI: http://dx.doi.org/10.1002/cplx.21656.

19. Fontes AMG, Guida HL, Barbosa JC, Antônio AMS, Cardoso MA, Garner DM, et al. Auditory stimulation with music intensifies cardiac autonomic responses to a mental task. Focus Alternative Compl Ther. 2014; 19(4): 198-207. DOI: http://dx.doi. org/10.1111/fct.12145.

20. Walsh TS, Ramsay P, Lapinlampi TP, Sarkela MO, Viertio-Oja $H E$, Merilainen PT. An assessment of the validity of spectral entropy as a measure of sedation state in mechanically ventilated critically ill patients. Intensive Care Med. 2008; 34(2): 308-15. DOI: http://dx.doi.org/10.1007/s00134-007-0858-x.

21. Wysocki M, Fiamma MN, Straus C, Poon CS, Similowski T. Chaotic dynamics of resting ventilatory flow in humans assessed through noise titration. Respir Physiol Neurobiol. 2006; 153(1): 54-65. DOI: http://dx.doi.org/10.1016/j.resp.2005.09.008.

22. Ponnusamy A, Marques $J$, Reuber M. Comparison of heart rate variability parameters during complex partial seizures and psychogenic nonepileptic seizures. Epilepsia. 2012; 53(8): 131421. DOI: http://dx.doi.org/10.1111/j.1528-1167.2012.03518.x

23. Abásolo D, Hornero R, Espino P, Alvarez D, Poza J. Entropy analysis of the EEG background activity in Alzheimer's disease patients. Physiol Meas. 2006; 27(3): 241-53. DOI: http://dx.doi. org/10.1088/0967-3334/27/3/003.

24. Shannon CE. A mathematical theory of communication. ACM Sigmobile Mobile Comp Comm Review. 2001; 5(1): 3-55. DOI: http://dx.doi.org/10.1145/584091.584093.

25. Hornero R, Abásolo D, Escudero J, Gomez C. Nonlinear analysis of electroencephalogram and magnetoencephalogram recordings in patients with Alzheimer's disease. Philos Trans A Math Phys Eng Sci. 2009; 367(1887): 317-36. DOI: http://dx.doi. org/10.1098/rsta.2008.0197.
26. Anderson TW, Darling DA. A test of goodness of fit. J Am Statist Assoc. 1954; 49(268): 765-9. DOI: http://dx.doi.org/10.1080/01 621459.1954.10501232.

27. Ryan Jr TA, Joiner BL. Normal probability plots and tests for normality. [cited 2014 July 03] Available from: http://www. minitab.com/uploadedFiles/Content/News/Published Articles/ normal probability plots.pdf.

28. Razali NM, Wah YB. Power comparisons of shapiro-wilk, kolmogorov-smirnov, lilliefors and anderson-darling tests. J Statist Mod Analytics. 2011; 2(1): 21-33.

29. Jolliffe I. Principal component analysis. Wiley Online Library; 2005. DOI: http://dx.doi.org/10.1002/0470013192.bsa501.

\section{Comment on this article:}

\section{$4[8$ in $8+s$.}

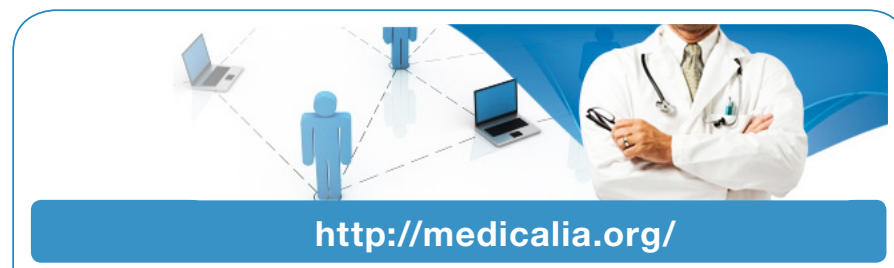

Where Doctors exchange clinical experiences, review their cases and share clinical knowledge. You can also access lots of medical publications for free. Join Now!

\section{Publish with iMedPub}

http://www.imed.pub

International Archives of Medicine is an open access journal publishing articles encompassing all aspects of medical science and clinical practice. IAM is considered a megajournal with independent sections on all areas of medicine. IAM is a really international journal with authors and board members from all around the world. The journal is widely indexed and classified Q1 in category Medicine. 